\title{
Estudio sobre la colaboración inter-institucional en procesos de innovación pública
}

\author{
Pablo Galaz \\ Agencia de Cooperación Internacional de Chile
}

\begin{abstract}
Resumen
El presente artículo profundiza en el rol de la colaboración inter-institucional en procesos de innovación pública, a través del estudio de dos casos: el primero relacionado con la gestión de pacientes con pie diabético en una sub-red de salud, y el segundo en torno a la administración de tres piscinas municipales temperadas. Como conclusiones del trabajo se identifican cinco puntos claves para gestionar procesos colaborativos de innovación, los cuales son (i) identificar si se requiere innovar a través de un trabajo colaborativo, (ii) disponer de equipos de trabajo que aborden los desafíos a resolver, (iii) garantizar el cumplimiento de roles mínimos necesarios para gestionar la innovación colaborativa, (iv) promover espacios de colaboración donde se privilegie la gestión de conocimiento tácito por sobre el explícito, y (v) buscar evidencia de los resultados obtenidos a partir de las soluciones implementadas.
\end{abstract}

Palabras clave: Innovación pública, colaboración inter-institucional.

\section{Study on interinstitutional collaboration in public innovation processes}

\begin{abstract}
This article deepens on the role of inter-institutional collaboration in processes of public innovation, through the study of two cases: the first related to the management of patients with diabetic foot in a health sub-network, and the second about the administration of three municipal heated swimming pools. On the findings of this work five key points are identified to manage collaborative innovation processes. They are the following: (i) identify if it is required to innovate by collaborative work, (ii) have teams that address the challenges to solve, (iii) ensure compliance with minimum roles needed to manage collaborative innovation, (iv) promote collaborative spaces where the management of tacit knowledge is privileged over the explicit and (v) look for evidence of the results obtained from the solutions implemented.
\end{abstract}

Keywords: Public innovation, inter-institutional collaboration.

\section{Colaboración inter-institucional en procesos de innovación pública}

La innovación pública se ha posicionado como una línea de acción para mejorar la performance del Estado, a través de la materialización y funcionamiento de nuevas ideas que creen valor público (Mulgan, 2007). Sin ir más lejos, algunos autores plantean que la gestión sistemática de la innovación, al igual que la gestión de recursos humanos, gestión presupuestaria, o planificación estratégica,

*Dirección de correspondencia [Correspondence address]:

Pablo Galaz, Universidad de Chile

E-mail: pgalaz@dii.uchile.cl debiese ser una disciplina central y esencial de cualquier organización pública (Bason, 2010; Eggers y Kumar, 2009).

$\mathrm{Al}$ respecto, la literatura internacional (Bason, 2010; Behn, 2008; Bloch y Bugge, 2013; European Commission, 2013; Sørensen y Torfing, 2011) da cuenta de una tendencia reciente por estudiar la gestión de procesos de innovación pública en base a una estrategia colaborativa, la cual busca construir soluciones integradoras entre actores que enfrentan problemas comunes (Hartley, 2008).

Lo anterior no es fácil, considerando que en la arena pública interactúan diversos actores. Así, la colaboración puede darse de múltiples formas: entre unidades al interior de una misma institución (intra-institucional), entre distintas instituciones públicas (inter-institucional), entre instituciones públicas, privadas y tercer sector; y con la 
ciudadanía (Eggers y Kumar, 2009; Hartley et al., 2013).

En este contexto, el presente trabajo se acota a estudiar de manera exploratoria los procesos de innovación pública colaborativa inter-institucional, con el fin de generar recomendaciones que contribuyan a la innovación pública, buscando: (i) que instituciones públicas de similar función (como municipios o consultorios) puedan homogenizar tanto sus procesos internos como la provisión de bienes y servicios públicos; y (ii) que instituciones públicas de distintos niveles jerárquicos y sectores que se encuentren interrelacionados (como consultorios con hospitales) mejoren la cadena de valor público donde interactúan.

\section{Innovación, proceso y valor público}

Los trabajos que han explorado el estado del arte sobre innovación pública (De Vries et al., 2015; Kattel et al., 2013) señalan que este concepto está asociado a nuevas soluciones, distintas a las ya existentes, que son implementadas por y/o para el sector público a fin de generar valor público.

Ahora bien, estas soluciones no ocurren de manera espontánea. La literatura internacional ha tendido a ampliar el concepto, promoviendo la innovación como un proceso que parte con la definición de un problema, seguido de la generación y selección de ideas, que posteriormente requieren ser testeadas mediante prototipos, para luego implementarlas, evaluarlas, difundirlas, y escalarlas en la medida de lo posible (Mulgan y Albury, 2003; Eggers y Kumar, 2009; Bason, 2010; Hartley et al., 2013).

La importancia de entender la innovación como un proceso radica en que una buena idea es solo el comienzo (Eggers y Kumar, 2009), y una parte importante del trabajo se juega en las fases de implementación y gestión del cambio. Para que esto tenga sentido en el sector público, se requiere de un Estado dotado institucionalmente con habilidades, recursos, métodos organizacionales, liderazgo y cultura (Mulgan y Albury, 2003), y que sea capaz de generar y seleccionar las mejores ideas posibles, implementarlas efectivamente y garantizar la creación de valor público (Bason, 2010).

\section{Complejidades para innovar en el sec- tor público}

Adicional a la complejidad inherente que tiene cualquier proceso de cambio, en el sector público se suman, o más bien se multiplican y potencian, las restricciones políticas, la necesidad de realizar estos cambios a la vista de la ciudadanía y la prensa, presiones externas, múltiples stakeholders con agendas contradictorias, presiones de sectores políticos por proteger los cargos de sus afiliados cualquiera sea su desempeño, dificultades en la modificación de normas y leyes, rigideces presupuestales y administrativas, o en el peor caso, capturas institucionales difíciles de romper (Waissbluth, 2008).

$\mathrm{Al}$ respecto, la literatura menciona recurrentemente elementos que constituyen el denominado ADN anti-innovación del sector público (Bason, 2010; Bloch y Bugge, 2013; IDEO, 2011), y que hacen de la innovación pública un fenómeno con claras diferencias respecto a la innovación privada y, en menor medida, con la innovación social (Kattel et al., 2013). Algunos de estos elementos son los siguientes.

- Los sistemas y estructuras del Estado están diseñados para funcionar de manera fiable, no para adaptarse a contextos cambiantes.

- Los ciclos políticos llevan a los policy makers a buscar "soluciones rápidas", las cuales no siempre son sustentables en el largo plazo y que no se hacen cargo de las causas reales de los problemas públicos.

- La burocracia y desagregación de funciones y roles impiden realizar acciones de manera coordinada. Si bien se puede dar dentro de una misma organización, este aspecto se hace más evidente cuando se requiere la coordinación de dos o más instituciones para resolver un problema de manera efectiva.

- La sub-estimación de las capacidades organizacionales que realmente se requieren para ejecutar proyectos innovadores y cumplir con el objetivo original.

- La escasa definición de procesos e instancias para que funcionarios públicos puedan proponer, probar y explorar nuevas ideas.

- La escasa integración de la ciudadanía en el diseño de nuevos bienes o servicios públicos, obteniéndose ideas que responden solamente al interés y voluntad de directivos y, en algunos casos, funcionarios públicos.

- La poca capacidad para realizar mediciones efectivas de resultados, haciendo imposible justificar presupuesto para investigación y desarrollo.

- El bajo conocimiento sobre cómo escalar innovaciones, impidiendo saber dónde centrar los esfuerzos y generar valor público. 
- La alta aversión al riesgo que existe dado que se presume que el fracaso en el desarrollo de una innovación puede considerarse desde una ineficiencia en la ejecución presupuestaria (uso de recursos público mal gastados), así como costos políticos de alto impacto (dudosa calidad de los resultados obtenidos en base a la promesa de valor establecida).

De este modo, es clave entender que cualquier proceso de innovación dista de ser claro y lineal. Al contrario, es un proceso complejo e iterativo (Hartley et al., 2013), y donde la secuencia a seguir no siempre se da de manera fluida y clara (Brown, 2009). Esto, por cierto, se acentúa en las etapas tempranas de innovación, las cuales tienen un efecto en el desarrollo y éxito de soluciones innovadoras (Gassmann y Schweitzer, 2014; Verworn, 2009).

\section{Fuzzy front end de la innovación}

Un concepto bastante enriquecedor para comprender la innovación pública como proceso, es lo que la Teoría del New Product Development define como fuzzy front end (FFE) de la innovación. $\mathrm{Su}$ traducción más próxima es frontera temprana y difusa, y hace alusión al conjunto de actividades que transcurren entre el momento en que se visualiza una oportunidad de innovar, y el instante desde el cual se disponen recursos focalizados para el desarrollo estructurado de la innovación en cuestión (Gassmann y Schweitzer, 2014; Gaubinger y Rabl, 2014; Herstatt y Verworn, 2001; Koen et al., 2001; Reid y de Brentani, 2004).

La FFE contempla el desarrollo de actividades en un contexto incierto, ambiguo y complejo que requiere equilibrar los insumos y factores catalizadores del proceso de innovación en combinaciones, no-lineales a fin de garantizar la deseabilidad, viabilidad y factibilidad de una innovación (Mootee, 2011).

Lo peor que puede ocurrir en la FFE es comenzar con una solución pre-definida, y luego buscarle un problema. Al contrario, el proceso de innovación debe partir identificando problemas, o bien reconociendo oportunidades de mejora (Bason, 2010). Lo anterior no es trivial, ya que se requiere que las organizaciones destinen tiempo y recursos para habitar en el FFE de la innovación, a fin de observar, entender y definir problemas de manera correcta (Kim y Wilemon, 2010).

Asimismo, es de suma relevancia generar certeza y convicción suficiente para que las ideas que surjan en la FFE puedan ser implementadas con posterioridad. Lo anterior está condicionada por el nivel de incertidumbre que tenga la organización, en particular la cúpula directiva, sobre factores tecnológicos, de entorno, recursos requeridos, capacidades y barreras de las organizaciones, entre otros (Kim y Wilemon, 2010).

\section{Innovación colaborativa}

La innovación no es algo nuevo para el sector público, siendo posible distinguir estrategias que suelen utilizarse en procesos de innovación pública, como lo son aquellas basadas en la línea del New Public Management (NPM), o bien un enfoque neo-weberiano (Hartley et al., 2013).

Sin perjuicio de lo anterior, la literatura internacional reciente (Bason, 2010; Behn, 2008; European Commission, 2013; Sørensen y Torfing, 2011) da cuenta de una tendencia clara por estudiar la gestión de procesos de innovación pública en base a una estrategia colaborativa. Esta estrategia promueve la construcción de soluciones integradoras entre actores que enfrentan problemas comunes y que afectan diversos niveles y sectores (Hartley et al., 2013). Dicha estrategia, a diferencia de la basada en el NPM y el enfoque neo-weberiano, no se funda en una teoría específica, sino que en múltiples y emergentes experiencias internacionales, las cuales han sido abordadas y estudias en profundidad de manera reciente (Hartley et al., 2013).

Cada día se requiere desarrollar nuevos paradigmas que hagan sentido a gestores públicos para enfrentar nuevos desafíos complejos y transversales, lo cual requiere pensar en las múltiples interconexiones, interdependencias e interacciones que se dan en el sector público (Benington y Moore, 2011).

Considerando que en la arena pública interactúan diversos actores, la colaboración puede darse de múltiples formas: entre unidades al interior de una misma institución (intra-institucional), entre distintas instituciones públicas (inter-institucional), entre instituciones públicas, privadas y tercer sector; y con la ciudadanía (Eggers y Kumar, 2009; Hartley et al., 2013).

\section{Innovación y colaboración inter- institucional}

Como ya se mencionó, la colaboración interinstitucional es una de tantas formas que tiene la innovación colaborativa. Esta hace referencia a procesos colaborativos donde dos o más institución públicas pueden verse afectadas por el mismo problema, aunque de manera distinta. En este nivel, existen 
dos tipos de colaboración inter-institucional.

Por un lado está la colaboración entre pares, donde instituciones públicas que cumplen funciones idénticas o similares (como municipios, consultorios, escuelas públicas, etc.) colaboran para homogenizar tanto sus procesos internos como la provisión de bienes y servicios públicos en base a al conocimiento pre-existente en ámbitos comunes.

Por otra parte, existe la colaboración entre niveles jerárquicos y sectores, donde instituciones públicas que tienen dependencias jerárquicas (como hospitales con consultorios), o bien trabajan en espacios comunes (como colegios con consultorios), buscan asociarse para generar innovaciones que requieran de una coordinación mayor.

Estos contextos no son fáciles de abordar, en cuanto se trata de instituciones con historias y contextos propios. Por tanto, más que una guía sobre cómo gestionar este tipo de procesos, lo siguiente son recomendaciones extraídas de la literatura internacional para llevar a cabo este proceso de la mejor manera posible.

\section{Recomendaciones para gestionar la innovación colaborativa inter- institucional}

En primer lugar, se ha de ser conscientes de que la experiencia y el conocimiento de otras instituciones públicas, sin duda, son una fuente primaria de inspiración para innova (Farah y Spink, 2008; Bloch y Bugge, 2013). De no tomarse en cuenta este principio, entonces es altamente probable que se cometan errores que otros ya vivieron.

Segundo, un elemento a considerar es el tipo de conocimiento que se desea gestionar. Típicamente, el conocimiento ha sido divido en dos categorías: conocimiento explícito y conocimiento tácito (Denner y Diaz, 2011). El conocimiento tácito es intangible, y se conecta a perspectivas personales, intuiciones, emociones, creencias, know-how, experiencias, valores, expertise y habilidades. Por sus características resulta difícil de formalizar y comunicar. El conocimiento explícito, por su parte, corresponde a aquel que puede ser articulado, codificado y almacenado en algún medio como manuales, documentos y procedimientos, haciéndolo factible de enviar o transmitir de manera "instantánea" a otros.

De estos, los actores que participan de un proceso de innovación colaborativo debiesen privilegiar el conocimiento tácito por sobre el explícito. En efecto, la forma más tradicional para gestionar el conocimiento tiene relación con estructurar y for- malizar experiencias exitosas en el sector público para que ellas puedan ser replicadas. Lo anterior se ha hace frecuentemente mediante concursos de ideas, concursos de proyectos, premios a innovaciones ya ejecutadas, y/o repositorios (o bancos) de buenas prácticas en sitios web. Dichas iniciativas pueden ser categorizadas como estrategias de "codificación" (Hansen, Nohria \& Tierney, 1998; en Denner y Diaz, 2011). Este enfoque busca que el conocimiento madure y se transforme en productos estandarizados con información explícita.

No obstante, Hartley (2008) muestra evidencia a partir de la experiencia Beacons Scheme $^{1}$ de que los métodos del tipo "cara-a-cara" (por ejemplo, días abiertos y visitas guiadas) son más efectivos para el aprendizaje inter-organizacional en contraste a actividades vinculadas a la transferencia de información "empaquetada" (como videos, CD y páginas web). Este tipo de estrategia de gestión del conocimiento, denominada como estrategia de "personalización", privilegia la obtención de productos personalizados a través de procesos de adaptación donde prevalece el intercambio de conocimiento tácito (Hansen, Nohria \& Tierney, 1998; en Denner y Diaz, 2011).

Por otra parte, Behn (2008) destaca la necesidad de contar con más de una sola instancia de tipo "cara-a-cara" para el intercambio de experiencia y conocimiento. Tal como se señaló antes, el proceso de innovación dista de ser un proceso claro y lineal, siendo por lo general bastante complejo, iterativo y difuso.

Por último, se promueve contar con roles que permitan nutrir y mantener este proceso "a toda máquina" (Behn, 2008). En esta línea, es recomendable contar con roles específicos para gestionar procesos de innovación colaborativa (Centro de Sistemas Públicos, 2015; Hartley et al., 2013).

- Convocante. Motivar, empoderar y lograr juntar a actores, creando y desarrollando la "arena de interacción", poner la agenda, aclarar el proceso y promover el ajuste de expectativas.

- Mediador. Tener claridad de las interdependencias, gestionar el proceso de manera segmentada, y construir confianza y resolver disputas alineando intereses.

- Catalizador. Vincular nuevo conocimiento y a otros actores interesados, explorar restricciones y oportunidades existentes y emergentes, promover el pensamiento "fuera de la caja".

\footnotetext{
${ }^{1}$ Programa nacional desarrollado en el Reino Unido, diseñado para reconocer y premiar la innovación y excelencia en gobiernos locales a partir de experiencias exitosas.
} 
- Gestor del cambio. Hacer que las ideas generadas y seleccionadas, se implementen de la mejor manera posible. Esto implica dar seguimiento al proceso completo.

Por otra parte, también deben considerarse las siguientes recomendaciones basadas en el documento "Colaboración Pública: Manual Metodológico para la Innovación en el Sector Público" (Centro de Sistemas Públicos, 2015)2.

En primer lugar, todo espacio de colaboración debe ser programado por quien esté liderando el proceso, para lo cual se deben considerar el objetivo del encuentro, la revisión de acuerdos y compromisos anteriores, participantes (al menos el equipo inicial), fuentes de información disponibles, lugar del encuentro (espacio físico), duración cronológica, y elaboración de un acta del encuentro.

Segundo, sea cual sea el espacio que se diseñe, éste debiese considerar dos momentos al menos. Por un lado se recomienda que exista un momento de divergencia, en el cual exista dedicación exclusiva para divergir en el análisis de información, exploración de oportunidades, generación de ideas, aclaración de ciertas temáticas que no son del todo conocidas, etc. Se debe tener claridad sobre la duración que tendrá este momento, el cual no debe ser inferior a la mitad del tiempo programado para cada espacio. Por otro lado, se debe considerar un momento de convergencia, cuyo fin es converger a acuerdos (acciones que son consensuadas por los equipos de trabajo, aplicables a todos quienes participen de futuros espacios relacionados al problema identificado) y compromisos (acciones que tienen un responsable concreto, y que debe ser cumplido en una fecha establecida).

En tercer lugar, las actividades de co-creación son fundamentales para que el proceso de innovación colaborativa pueda darse de manera consistente. Todas ellas se encuentran fuertemente influenciadas por el concepto de co-creación, lo cual puede ser interpretado como el diseño de nuevas soluciones realizado por sus mismos usuarios, y no para ellos (Bason, 2010). Así, toman relevancias encuentros como los Círculos de Innovación, las visitas abiertas y guiadas, y las reuniones de seguimientos, todas aplicadas en los casos estudiados.

Lo anterior puede llevar a que el equipo de trabajo redefina el problema, acote el desafío, integre nuevos actores al equipo, y lleve a cabo otras actividades de $\mathrm{CO}^{-}$-creación. La iteración a esta altura puede durar varias semanas o meses, dependiendo

\footnotetext{
${ }^{2}$ Este documento ha sido elaborado a partir de los resultados del Proyecto "Colaboración Pública" (ver sección 3.1.1 Proyecto FONDEF "Colaboración Pública”).
}

de la envergadura de los desafíos y soluciones. Sin embargo, es clave que el trabajo converja a una cartera de soluciones deseables, factibles y viables de implementar.

\section{Metodología}

\section{Fuente de información: Proyecto "Co- laboración Pública"}

Los casos abordados en el presente trabajo corresponden a experiencias de innovación colaborativa estudiadas durante la fase de experimentación del Proyecto D10I 1034 "Sistema de Gestión del Conocimiento para la Transferencia de Innovaciones y Buenas Prácticas de Gestión en Gobiernos Locales" (también denominado Proyecto "Colaboración Pública"), seleccionado en el XVIII Concurso I $+\mathrm{D}$ del Fondo de Fomento al Desarrollo Científico y Tecnológicos del Consejo Nacional de Investigación Científica y Tecnológica. Su duración fue de 40 meses (diciembre 2012-abril 2015) y contó con el apoyo institucional de la Subsecretaría de Desarrollo Regional y Administrativo, Ministerio de Salud, y la Asociación Chilena de Municipalidades.

Dicho proyecto tenía por objetivo diseñar una institucionalidad, procesos y sistemas para gestionar el conocimiento entre municipios y otras entidades locales (como consultorios), a fin de disminuir la heterogeneidad que existe en la provisión de bienes y servicios públicos que entregan dichas instituciones a la población.

Para ello, durante la fase de experimentación se testearon dos estrategias metodológicas. La primera se focalizaba en la transferencia de buenas prácticas, lo cual supone documentar experiencias exitosas, buscar otra entidad interesada y apoyar la implementación. Pese a que se documentaron 61 prácticas en una plataforma web enfocada a la atención primaria de salud, en 18 meses solo se logró transferir 1 de ellas en un solo consultorio. Por tanto, se descartó esta metodología como una estrategia exitosa para cumplir el objetivo del proyecto.

La segunda metodología puso sus esfuerzos en la co-creación de innovaciones, a través de la conformación de redes de trabajo colaborativas que trabajan principalmente de manera presencial (cara-acara) en la generación de nuevas ideas y su posterior implementación. 


\section{Selección de casos a estudiar}

Se seleccionaron dos de los seis casos de éxito obtenido bajo la metodología de co-creación de innovaciones, según los siguientes criterios.

- Evidencia de logros y resultados concretos. Si bien en los seis casos existe evidencia de cambios implementados, se seleccionaron aquellos donde se registra información, al menos postintervención, para estimar los efectos de las intervenciones.

- Disponibilidad y acceso a información del proceso. Se consideran casos donde el autor del presente trabajo participó directamente durante la ejecución del Proyecto "Colaboración Pública".

- Variedad del fenómeno. Los casos abordados durante el Proyecto "Colaboración Pública" contempla dos tipos de colaboración interinstitucional: entre partes, y entre niveles jerárquicos. De esta forma, se seleccionan un caso de cada tipo.

Bajo estos criterios, los casos seleccionados corresponden a dos experiencias de innovación colaborativa.

- Colaboración en Sub-Red de Salud Pública La Florida en temas de pie diabético

- Colaboración entre administraciones de piscinas municipales temperadas

\section{Estructura de los casos de estudio y elementos transversales}

Los casos se presentan tomando como referencia la metodología Colaboración Pública (Centro de Sistemas Públicos, 2015), la cual busca representar procesos de innovación pública colaborativa. De este modo, los casos se presentan bajo la siguiente estructura.

- Fase temprana y difusa de la innovación. En particular, se relata lo relacionado a (i) la comprensión del problema y definición del desafío a abordar, y (ii) la conformación del equipo y co-creación de innovaciones.

- Fase de implementación. Específicamente, se describe (i) el cumplimiento de compromisos y testeo de innovaciones, y (ii) la evaluación preliminar de las acciones realizadas.
Acorde a lo señala por Behn (2008), la fase de experimentación del Proyecto "Colaboración Pública" consideró la participación de un grupo interdisciplinario de profesionales denominado Equipo Colaboración Pública. La labor de este equipo fue transversal a todos los casos, cuyo integrantes cumplían los roles básicos que demandan los procesos de innovación colaborativa: convocantes, mediadores, catalizadores, y gestores de cambio (Centro de Sistemas Públicos, 2015; Hartley et al., 2013).

Por otra parte, los casos estudiados consideran la realización de las siguientes actividades, la cuales requieren ser descritas para una mayor comprensión.

- Círculos de Innovación. Un círculo de innovación es un espacio de diálogo abierto donde se comparten experiencias y conocimiento respecto a un desafío común. En estos espacios se busca abordar un desafío de manera escalonada, generando mejoras e innovaciones incrementales que permitirían resolver poco a poco el desafío en cuestión. Como producto de ello es alcanzar acuerdos y compromisos que permitan delinear una línea clara de trabajo en el corto plazo.

- Visitas abiertas y guiadas. Estas permiten comprender el contexto donde se han implementado experiencias exitosas, y detectar los factores claves de éxito para la adopción de prácticas.

- Reuniones de seguimiento. Una reunión de seguimiento corresponde a una instancia para evaluar el avance del trabajo que se ha realizado hasta el momento. Este tipo de espacios permite redefinir o precisar el desafío planteado inicialmente, así como considerar la integración de nuevos actores al proceso de innovación colaborativa. Vale decir, las reuniones de seguimientos permiten adaptar el trabajo colaborativo en función de la evolución que ha generado el proceso de co-creación. 


\section{Descripción de los casos de es- tudio}

\section{Colaboración en Sub-Red de Salud Pública La Florida en temas de pie diabético}

\section{Comprensión del problema y definición del desafío a abordar}

Las heridas de pie en pacientes diabéticos, condición denominada como pie diabético, son consecuencia de una diabetes de larga evolución y/o mal controlada. La insensibilidad de los pies es una de las complicaciones de la enfermedad y por ello aumenta el riesgo de heridas, y su posterior infección. Las consecuencias más graves para el paciente son la amputación y, excepcionalmente, la gangrena y muerte por infección. Asimismo, tanto la herida como la amputación implican aumento de la discapacidad y con ello repercusión en los años de vida saludable perdidos.

En Chile, cerca del 6,7\% de los pacientes con diabetes $(9,4 \%$ de la población nacional mayor a 15 años) ha tenido que consultar a un profesional de la salud en el último año por pie diabético. Asimismo, del total de pacientes con diabetes, el 48,3\% declara nunca haber asistido a algún tipo de examen de los pies (Ministerio de Salud, 2011). Adicionalmente, se estiman más de 2.000 amputaciones de pie por diabetes mellitus al año, lo cual podría reducirse hasta un $85 \%$ de implementarse estrategias de incluyan prevención, educación de pacientes y prestadores, tratamiento multidisciplinario de las úlceras y monitoreo estrecho (Seguel, 2013).

En este contexto, el proceso de innovación colaborativa se inicia con un diagnóstico realizado por la Unidad Técnica de Salud la Corporación Municipal de La Florida (UTS-COMUDEF). Dicha unidad estimó que el $50 \%$ de los pacientes que fueron atendidos el mes de enero de 2014 en algún Centro de Salud Familiar (CESFAM) de la comuna por problemas de pie diabético, también estaban inscritos en el Programa de Salud Cardiovascular $(\mathrm{PSCV})^{3}$. De ser significativo, esto implicaría que la mitad de los pacientes con pie diabéticos de la comuna tratan un síntoma del problema, y no sus casusas raíces. Por tanto, un desafío concreto pa-

\footnotetext{
${ }^{3} \mathrm{El}$ PSCV es una de las principales estrategias del Ministerio de Salud para contribuir a reducir la morbimortalidad asociada a las enfermedades cardiovasculares. Este ?nace? el año 2002 producto de la reorientación de los subprogramas de Hipertensión arterial (HTA) y Diabetes (DM), cuyo principal cambio fue incorporar el enfoque de riesgo cardiovascular global en el manejo de las personas bajo control, en lugar de tratar los factores de riesgo en forma separada.
}

ra la UTS-COMUDEF se vinculaba a la pregunta "¿cómo se podría incorporar a los pacientes con pie diabético al PSCV?".

A fin de levantar información histórica sobre pacientes atendidos por pie diabético, y comprarla con los registros del PSCV, durante el mes de marzo de 2014 el Equipo Colaboración Pública visita distintas Unidades de Apoyo $^{4}$ de los CESFAM (UACESFAM) en la comuna de La Florida. Lamentablemente, no fue factible acceder a esta información dado el temor de las encargadas de dichas unidades por infringir la Ley de Derechos y Deberes de los Pacientes $^{5}$, específicamente la restricción al acceso a información personal sin previa autorización de los pacientes.

No obstante, el contacto directo con las encargadas de las UA-CESFAM permitió detectar otro problema: la baja coordinación en el proceso de derivación de pacientes con pie diabético que requieren de atención especializada en el nivel secundario (hospitales). Efectivamente, en ese entonces existía poca claridad sobre el proceso de derivación de pacientes con pie diabético desde las UA-CESFAM de la comuna hacia el Policlínico de Pie Diabético del Hospital Clínico La Florida (PPD-HLF), inaugurado en enero de 2014. Esto producía, por ejemplo, que existiesen casos documentados de pacientes que, en vez de ser derivados al PPD-HLF, eran derivados a la Urgencia de dicho hospital, lugar donde se les postergaba la atención dada la priorización de otros pacientes, sin ser derivados internamente. De este modo, se identifica un nuevo desafío, relacionado a la pregunta "¿cómo se podría hacer más eficientes y efectiva la derivación de pacientes con pie diabético desde consultorios al hospital?".

\section{Conformación del equipo y co-creación de innovaciones}

Para abordar este desafío, el Equipo Colaboración Pública convoca y modera en el mes de abril de 2014 un Círculo de Innovación donde participan ocho personas provenientes de la UTS-COMUDEF (1 encargada), UA-CESFAM (4 encargadas), y del PPD-HLF (2 doctores especialistas y 1 enfermera).

En este encuentro se levantó el proceso general de derivación de pacientes con pie diabético desde una UA-CESFAM al PPD-HLF, a partir de lo cual se identificaron los siguientes puntos críticos.

\footnotetext{
${ }^{4} \mathrm{Al}$ interior de un CESFAM, la Unidad de Apoyo es la entidad responsable por la atención de pacientes con pie diabético.

${ }^{5}$ Ley $\mathrm{N}^{\circ} 20.584$ que regula los derechos y deberes que tienen las personas en relación con acciones vinculadas a su atención en salud. Para mayor información, visitar http://bcn.cl/1m0do [visto 25 de febrero de 2015]
} 
- Pedir una hora de atención de especialista cardiovascular en el PPD-HLF requería que un paciente entregase en dicho lugar, de manera presencial, la Solicitud de Interconsulta otorgada previo diagnóstico realizado en una UACESFAM.

- Encontrar la ubicación física del PPD-HLF al interior del mismo hospital era una labor compleja, por lo que algunos pacientes no sabían dónde entregar la Solicitud de Interconsulta y se perdían al interior del hospital.

- Programar una hora de atención de especialista cardiovascular en el PPD-HLF tardaba cerca de 5 días, contados desde la entrega física de la Solicitud de Interconsulta, pese a que una curación avanzada de herida de pie diabético debe iniciarse y realizarse en el nivel secundario de salud dentro de las 24 horas desde la confirmación de un diagnóstico desde algún establecimiento de $\mathrm{APS}^{6}$.

- No era posible para las UA-CESFAM saber si los derivados fueron atendidos en el PPDHLF, o bien cuando fueron dados de alta.

Para abordar estos puntos y resolverlos en el corto plazo, los participantes co-crearon un plan de acción conformado por dos simples acciones.

- Realizar una visita guiada por los profesionales del PPD-HLF al hospital, donde se muestre a las encargadas de las UA-CESFAM el recinto, a fin de que éstas puedan dar las indicaciones adecuadas a los pacientes derivados por pie diabético sobre dónde deben ser atendidos, evitando que estos se pierdan al interior.

- Crear un correo electrónico para testear la idea de que las UA-CESFAM puedan informar al PPD-HLF cuando un paciente sea derivado. Con ello se busca evitar que estos deban solicitar una interconsulta de manera presencial, y disminuir el tiempo que transcurre entre el momento que son derivados y reciben una hora de atención.

\section{Cumplimiento de compromisos y testeo de innovaciones}

Por un lado, en mayo de 2014 se concreta la visita guiada dentro del HLF comprometida en el Círculo de Innovación. En dicha instancia los médicos

${ }^{6}$ Para mayor $\quad$ información,
http://www.supersalud.gob.cl/difusion/572/w3-
propertyvalue-3130.html [visto 24 de febrero de 2015]

especialistas del PPD-HLF mostraron a las encargadas de las UA-CESFAM las instalaciones completas del hospital. De ese modo, además de conocer el policlínico, también se permite conocer los profesionales de Urgencias y de Radiología, ambas áreas estrechamente vinculadas al tratamiento de pacientes con pie diabético. Por otro lado, para testear la idea de que las UA-CESFAM puedan informar al PPD-HLF cuando un paciente sea derivado, se crea el correo electrónico piediabeticoHLF@gmail.com, al cual las UA-CESFAM deben enviar un correo electrónico ajuntando los documentos necesarios para que una enfermera del PPD-HLF pueda fijar una hora de atención con un especialista cardiovascular y confirmarla con el paciente. Estos documentos son los siguientes.

- Un formulario que contiene la información mínima requerida del paciente para fijar una hora de interconsulta (nombre paciente, teléfono de contacto, CESFAM donde es atendido, gravedad de las heridas del pie, entre otros elementos).

- Copia digital del registro físico de la Solicitud de Interconsulta emitida por el CESFAM (fotografía tomada con un celular, documento procesado con un scanner, etc).

De este modo, la UTS-CESFAM informó a todas las UA-CESFAM de la comuna sobre esta nueva forma para informar las derivaciones de pacientes de pie diabético al PPD-HLF, comenzando su operación el 1 de junio de 2014 .

\section{Evaluación de las acciones realizadas}

El correo piediabeticoHLF@gmail.com no solo fue utilizado como vía para solicitar interconsultas de pacientes con pie diabético, sino también para realizar consultas a los especialistas del PPDHLF sobre tratamientos, opiniones relacionadas a pacientes, evolución de los mismos, etc. En pocas palabras, se constituyó como una vía de comunicación ampliamente demandada por los CESFAM de la comuna de La Florida en temas relativos al problema del pie diabético.

Como factor clave para ello fue que, desde su implementación, la UTS-COMUDEF comunicará a todos los CESFAM de la comuna sobre el uso del correo. Vale decir, el testeo de esta nueva manera de derivar pacientes con pie diabético se aplicó de inmediato a un contexto real.

Para evaluar su funcionamiento, en noviembre de 2014 se analizaron las solicitudes realizadas hasta el 
31 de octubre de 2014, lo que representa los primero cinco meses de implementación. De dicha muestra, se da cuenta de lo siguiente.

- Se enviaron 61 casos de 8 UA-CESFAM. De este total, 11 corresponden a pacientes que fueron derivados a la Urgencia del hospital, y 50 a pacientes derivados al PPD-HLF.

- De este último grupo, el PPD-HLF tardó 0,7 días en promedio para responder a las UACESFAM, confirmando una hora de interconsulta. Asimismo, en promedio dichas horas eran fijadas para 4,3 días contados desde la recepción del correo.

A juicio de las encargadas de las UA-CESFAM, y el equipo del PPD-HLF, el plan de acción acordado fue de gran éxito, la creación del correo ha sido un gran aporte para mejorar la efectividad y eficiencia de la derivación de pacientes con pie diabético.

\section{Colaboración entre administraciones de piscinas municipales temperadas}

\section{Comprensión del problema y definición del desafío a abordar}

Entre los años 2012 y 2014, el Banco Integrado de Proyectos del Ministerio de Desarrollo Social registra 14 iniciativas de proyectos con recomendación favorable para la construcción de piscinas temperadas en Chile, tanto a nivel de diseño (4 proyectos) como ejecución (10 proyectos). El costo total de todos estos proyectos asciende a MM\$20.393 (en \$ 2014), donde la construcción de una piscina supera, en la mayoría de los casos, los MM\$2.000.

Usualmente, los municipios justifican la inversión en este tipo de recintos apelando a la carencia de una oferta de servicios deportivos de calidad para los habitantes de una comuna. Ahora bien, disponer de una oferta deportiva de calidad sostenida en el tiempo requiere manejar a cabalidad la especificidad que conlleva la operación y mantención de una piscina temperada. De no ser así, es altamente probable que estos recintos se transformen en un costo neto para los municipios con un funcionamiento intermitente.

Mejorar la gestión de piscinas temperadas es un desafío constante para los administradores de dichos recintos. Ello porque se deben considerar un gran número de aspectos, respondiendo preguntas tanto estratégicas como operacionales: ¿cuál es rol estratégico de la piscina para su municipio?, ¿cómo se cruza la demanda de los usuarios con la oferta deportiva de la comuna (pública y privada)?, ¿qué tipo de acciones se deben realizar para mantener adecuadamente la calidad del agua (principalmente temperatura, $\mathrm{pH}$ y nivel de cloro) y el funcionamiento los equipos (como motores, filtros, calderas, y deshumidificadores)?

\section{Conformación del equipo y co-creación de innovaciones}

Atendiendo al desafío de "¿cómo se podría mejorar la sustentabilidad operativa de las piscinas temperadas municipales?", el Equipo Colaboración Pública convocó y moderó un Club de la Innovación en octubre de 2013 entre los administradores de las piscinas temperadas administradas por los municipios de Providencia, Peñalolén y Santiago. Mientras que la primera administración llevaba diez años de operación a la fecha del encuentro, la segunda solo contaba dos años de funcionamiento, y la tercera se encontraba preparando el inicio de una marcha blanca y pronta inauguración.

El encuentro, casi en su totalidad, estuvo centrado en que los participantes pudiesen hablar libremente sobre el desafío planteado, declarándose interés por intercambiar conocimiento en los siguientes ámbitos.

- Infraestructura (piscinas, camarines, gimnasio, acumuladores de agua).

- Mantención del agua (volumen, tratamiento $\mathrm{pH}$, temperatura, cloro).

- Máquinas (bombas, filtros, deshumidificadores, calderas).

- Control de gestión (contratos de mantención, tarificación de servicios, venta y recaudación, gastos de aseo).

Para continuar con el trabajo colaborativo, los participantes se comprometieron en visitar presencialmente los recintos deportivos de los tres municipios participes de esta experiencia. Esto se justifica en cuanto una visita guiada permite comprender mejor el contexto donde se puede innovar, o bien donde ya se ha innovado y es factible adaptar soluciones. Para ello, el rol convocante del Equipo Colaboración Pública fue clave considerando la necesidad de consensuar la agenda de una administración que estaba ad-portas de abrir el recinto deportivo (Piscina Olímpica Temperada de Santiago), así como otras administraciones que operan de lunes a domingo en horario continuado prácticamente todo el día (Spa Club Providencia y Piscina Temperada de Peñalolén). 
Durante el mes de noviembre de 2013, se realizaron las tres visitas guiadas. Cada una fue dirigida por los responsables de los recintos en cuestión, así como con el personal vinculado a la mantención del equipamiento y agua. La duración promedio de cada instancia fue cercana a las 3 horas, permitiendo recorrer completamente las instalaciones de todos los recintos visitados (control de acceso, oficinas, camarines, sala de máquinas, piscinas, entre otros lugares).

Al finalizar cada encuentro se les solicitó a todos los participantes que evaluasen la experiencia, señalando si había cumplido con sus expectativas. En términos generales, esta modalidad de intercambio de conocimiento y experiencia fue altamente valorada ya que permitía emular la experiencia de los usuarios del recinto (acceso a la piscina, apreciación general del recinto, claridad del agua, temperatura, acústica del lugar, etc), así como las preocupaciones constantes de las administraciones (limpieza del recinto, mantención de temperatura y $\mathrm{pH}$ del agua, monitoreo del funcionamiento de máquinas como bombas, deshumidificadores, etc).

\section{Cumplimiento de compromisos y testeo de innovaciones}

Luego de realizar las visitas, el coordinador de la Piscina Temperada de Peñalolén declaró estar interesado en implementar mejoras basadas, principalmente, en la experiencia del Spa Club Providencia. La administradora de dicho recinto, por su parte, expresó su voluntad en apoyar a Peñalolén, pese a no tener interés en desarrollar mejoras en base a la experiencia de los otros participantes. Por su parte la administración de la Piscina Olímpica Temperada de Santiago declinó continuar trabajando colaborativamente ya que la inauguración del recinto deportivo estaba planificada para el mes de mayo de 2014 y estimaban que el tiempo dispuesto para terminar todos los temas pendientes era muy poco.

Para comenzar este trabajo, el Equipo Colaboración Pública junto al coordinador de la Piscina Temperada de Peñalolén trabajaron semanalmente durante los meses de diciembre de 2013 y marzo de 2014, levantando la situación actual del recinto, generando nuevas ideas y adaptando prácticas desde la experiencia de Providencia. Como producto de este trabajo se gatillaron soluciones en tres ámbitos.

- Planificación estratégica de servicios y tarifas. Junto con una nueva programación horaria de los servicios deportivos ofertados por recinto, centrada en las preferencias de los usuarios, se elaboró una metodología para estimar de mejor manera la tarifa asociada a cada curso y taller.

- Mantención de equipos. Se adaptó el método de mantención que se utilizaba en el Spa Club Providencia, realizándose una calendarización de revisiones preventivas junto con el diseño de un formulario de revisión para evaluar cada equipo a fin de tomar acciones en caso de detectarse falencias en la mantención.

- Mantención del agua. Se elabora un nuevo contrato con la empresa de mantención para aumentar el número de controles, se modifica el tipo de cloro que se utilizaba para mantener el agua, y se comienzan a utilizar nuevas herramientas para medir los niveles de $\mathrm{pH}$ y cloro del agua según los estándares de la SEREMI de Salud.

Todo lo anterior comienza a funcionar en el mes de abril de 2014, planificándose realizar una evaluación cinco meses después.

\section{Evaluación de las acciones realizadas}

Durante todo el período de implementación, el Equipo Colaboración Pública en su rol de gestor del cambio, realizó un seguimiento semanal con el coordinador la Piscina Temperada de Peñalolén para ir evaluando el cumplimiento de los compromisos. Por otro lado, el interés y la voluntad de la administración del Spa Club Providencia fueron fundamentales para acceder al conocimiento que han capitalizado en dicho recinto por más de una década.

Para evaluar el potencial efecto de las medidas tomadas, se estudiaron las renovaciones de planes deportivos de dos grupos de socios.

- Grupo pre-intervención. Socios que inscribieron por primera vez un plan deportivo durante el mes de agosto de $2013(n=277)$. Para este grupo, se estudiaron las renovaciones realizadas entre los mes de septiembre de 2013 y enero de 2014 .

- Grupo post-intervención. Socios que inscribieron por primera vez un plan deportivo durante el mes de abril de 2014 ( $n=277)$, Para este grupo, se estudiaron las renovaciones realizadas entre los mes de mayo de 2014 y septiembre de 2014.

A partir de este análisis se tienen los siguientes resultados. 
- El porcentaje de socios que renuevan su plan deportivo después del primer mes pasó de $54,6 \%$ (grupo pre-intervención) a 61,2\% (grupo post-intervención).

- El número de renovaciones promedio por socio durante los cinco meses posteriores a haberse inscrito en un plan deportivo pasó de 1,36 renovaciones (grupo pre-intervención) a 1,72 (grupo post-intervención), lo cual representa un aumento de un $26 \%$. En términos absolutos, esta variación implica que el grupo postintervención realizó 80 renovaciones más que el grupo pre-intervención en el período analizado.

- El porcentaje de socios que renovaron durante 5 meses consecutivos pasó de un 7,0\% (grupo pre-intervención) a $16,3 \%$ (grupo postintervención), lo que se traduce en una variación de un 132,9\%.

Cabe señalar que el grupo post-intervención estuvo sujeto a un aumento en el valor de cursos y talleres cercano al $10 \%$, lo cual fue estimado a través de la nueva metodología diseñada. Pese a ello, se observa que la demanda se mantuvo, e incluso se aprecia que tuvo una mayor tasa de fidelización, lo cual podría interpretarse como una mejor calidad del servicio. El Equipo Colaboración Pública estimó que las modificaciones tarifarias de los cursos, junto con los nuevos métodos para la mantención de máquinas y agua, se traducirían en beneficio para recinto cercano a MM\$60 (evaluación social a 5 años con una tasa de descuento de $6 \%$ ). Lo anterior se justifica en cuanto hay más ingresos y se realizarían menos re-inversiones en máquinas en el largo plazo.

\section{Conclusiones}

\section{¿Se requiere de colaboración inter- institucional para innovar?}

No todo problema puede y requiere ser resuelto a través de un proceso de innovación colaborativa. Para saber dónde es posible innovar de manera colaborativa, es necesario tener claridad absoluta del problema y poder identificar si hay espacios donde hace sentido establecer redes colaborativas para diseñar e implementar soluciones. Por tanto, antes de realizar una colaboración interinstitucional, es recomendable:

- Traducir el problema en uno o varios desafíos. Estos debiesen ser formulados de manera su- ficientemente amplia como para no sesgarse a ninguna solución pre-concebida, y debidamente acotada como para que existan límites que impidan perderse durante el proceso de innovación. Hay que recordar que los procesos de innovación consideran una fase bastante difusa en la cual es fácil perder el rumbo.

- Identificar aquellos desafíos donde se requiere de trabajar colaborativamente. De los distintos tipos de colaboración que pueden establecerse para innovar en el sector público, la colaboración inter-institucional es recomendable cuando un desafío requiere y puede ser abordado por una o más instituciones públicas. Esto ocurre, por ejemplo, cuando se desea homogenizar la provisión de un bien o servicio público, así como cuando mejorar la cadena de valor público implica la coordinación inter-institucional.

En los dos casos estudiados, el problema que motivó el trabajo colaborativo fue traducido a desafíos que, si bien no buscan resolver el problema completo, sí permiten focalizar el trabajo y obtener resultados. Cabe destacar el caso de colaboración en la Sub-Red de Salud La Florida, donde se identificaron dos desafíos en torno al mismo problema, y de los cuales el desafío trabajado ("¿cómo se podría hacer más eficientes y efectiva la derivación de pacientes con pie diabético desde consultorios al hospital?") tenía más sentido ser abordado de manera colaborativa respecto el establecido inicialmente ("¿cómo se podría incorporar a los pacientes con pie diabético al PSCV?").

Asimismo, el Equipo Colaboración Pública tuvo una gran influencia en acotar los desafíos trabajados en cada caso, a fin de que fuesen factibles de abordar por un grupo de instituciones públicas.

\section{Tamaño, experiencia y capacidad re- solutiva en los equipos de trabajo}

Si el desafío a abordar requiere trabajar colaborativamente entre distintas instituciones públicas, entonces es necesario conformar al menos un equipo de trabajo que lo aborde. Para su conformación, se recomienda respetar y equilibrar los siguientes factores.

- Número de participantes. Hay que considerar que un número muy grande de participantes puede condicionar la convocatoria, y dificultar la moderación al momento de trabajar.

- Experiencia y conocimiento. Para maximizar la divergencia de ideas y variabilidad de conocimiento, deben considerase funcionarios que 
representen múltiples visiones sobre el mismo desafío y cuyos trabajos se vinculen directamente con éste. Con esto se espera que todos los participantes puedan hablar el "mismo lenguaje", así como empatizar por el "trabajo del otro".

- Capacidad para tomar decisiones. Entre los participantes se debe considerar la incorporación de actores que puedan decidir si una innovación puede (o no) ser implementada. De no contar con esto, entonces es probable que las ideas generadas queden solo en eso: ideas.

- Cercanía geográfica. La cercanía geográfica entre las instituciones que conforman un equipo de trabajo constituye un elemento de diseño clave a la hora de poner en marcha un proceso de innovación pública inter-institucional. Esto se funda por dos motivos: (i) existe mayor probabilidad de que las instituciones compartan contextos similares para abordar las mismas problemáticas, y (ii) facilita la convocatoria y participación del equipo de trabajo en espacios de colaboración donde el "cara-a-cara" es fundamental.

En los casos estudiados, la conformación de los equipos de colaboración fue labor del Equipo Colaboración Pública, respetándose cada uno de los criterios señalados.

Pese a que no se evidenció en los casos estudiados, debe recordarse que los procesos de innovación colaborativa no están exentos a procesos de captura, e incluso a dinámicas de poder donde actores pueden focalizar en imponer sus soluciones. Por tanto, este también es un factor que debe ser considerado al momento de la conformación de los equipos de trabajo.

\section{Cumplir con los roles mínimos}

El Equipo Colaboración Pública fue clave al momento de convocar y moderar encuentros de diseño e intercambio de experiencias, así como en catalizar el proceso y gestionar el cambio.

En los dos casos estudiados, se aprecia que cada rol se intensifica, o atenúa, a medida que el proceso avanza. Inicialmente, el rol "convocante" fue fundamental para conformar los primeros equipos de trabajo. Luego, el rol "moderador" permitió construir lazos de confianza entre los participantes a fin de integrar la mirada de todos en la definición del desafío y generación de ideas. Posterior a ello, el rol "catalizador" permitió, en ambos casos, disponer de resultados en el corto plazo que validaban el trabajo del equipo. Por último, el rol "gestor del cambio" permitió que darle seguimiento y continuidad para que la implementación de las ideas generadas no se perdiese en la rutina y cotidianeidad.

$\mathrm{Al}$ respecto, es completamente recomendable buscar apoyo "fuera de la caja" si se aprecia que algunos de estos roles no pueden ser cumplidos por el equipo de trabajo que se conforme. En los casos estudiados, los equipos de trabajos carecían de las competencias y/o conocimientos necesarios para cumplir con los roles mínimos que demanda una proceso de innovación de tipo colaborativo, por lo cual el Equipo Colaboración Pública tuvo que tomarlos.

\section{Gestión del conocimiento tácito y es- pacios de colaboración}

El rol que tiene el conocimiento tácito en los procesos de innovación colaborativa es gravitante. La metodología para estructurar el trabajo colaborativo debe fundarse en actividades del tipo "cara-acara", donde sea posible establecer conversaciones (conocimiento tácito), y no acotarse a compartir información "empaquetada" (conocimiento explícito) en formato de manuales, CD, videos, audios $\mathrm{u}$ cualquier otro equivalente.

Favorecer espacios de intercambio de conocimiento de tipo presencial permite que los participantes del proceso puedan presentarse y conocerse de una manera que no se daría si la única oportunidad de conocer "al otro" es descargando y leyendo un documento. Lo anterior, facilita, aunque no garantiza, que se generen vínculos de confianza entre los participantes que trabajarán de manera colaborativa. Sin ello, es prácticamente imposible identificar y consensuar cuál es problema, ya que existen diversas interpretaciones sobre "lo que no funciona" y "lo que falta".

Ahora bien, trabajar colaborativamente en base a encuentros presenciales es mucho más costoso que difundir experiencias de manera abierta a través de plataformas virtuales. Más aún cuando se pretende que la colaboración ocurra entre servicios públicos que se encuentran ubicados a grandes distancias. Por tanto, aquí entra en juego el trade-off entre el costo de generar espacios de interacción presencial y los potenciales de mejoras que se pueden obtener. 


\section{Búsqueda de evidencia y resultados}

Finalmente, un punto a destacar es la búsqueda de evidencia y resultados. Este punto, por cierto, incide en todo proceso de innovación, sea o no colaborativo.

Para evaluar el impacto de una intervención se requiere de información que dé cuenta sobre el cumplimiento que ha tenido la innovación durante un período de tiempo pre-establecido respecto los objetivos que se fijaron para él. En este contexto, existe cinco niveles que pueden ser identificados para evidenciar el impacto de una innovación (Puttick y Ludlow, 2013).

- Nivel 1. Se describe la innovación, señalando por qué ella importa, de manera lógica, coherente y convincente.

- Nivel 2. Se cuenta con datos que muestran un cambio positivo luego de materializada una innovación, pero no permiten confirmar si ello es producto de la misma.

- Nivel 3. Se demuestra una relación causal entre el impacto y la innovación implementada a través del uso de grupos de control o de comparación.

- Nivel 4. Es posible disponer de una o más evaluaciones independientes que confirmen las conclusiones obtenidas.

- Nivel 5. Se dispone de manuales, sistemas y procedimientos que garantizan un impacto positivo de manera consistente.

En este punto, el principal desafío guarda relación con disponer datos fidedignos que permitan argumentar, de manera razonable (e incluso, científica), que la innovación alcanzada fue positiva. Para los casos descritos, los desafíos definidos requerían ser resueltos de manera rápida, razón por la cual no hubo tiempo para establecer una línea base clara a fin medir el efecto de los cambios.

\section{Recomendaciones}

\section{Implicancias para el diseño de políti- cas públicas}

Por un lado, una comprensión acabada sobre la colaboración inter-institucional puede contribuir al diseño de programas en el sector público, como premios a innovaciones efectivas, o bien bancos de buenas prácticas, para ir más allá de la difusión y lograr un aprendizaje efectivo entre los organismos que participen.

En Chile, este tipo de iniciativas han abundado desde la década de los 90`s, con poca duración, y sin resistir a cambios de administración. Ello hace pensar en la importancia no solo de aspectos metodológicos para garantizar su efectividad, sino también en una gobernanza y soporte institucional a fin de maximizar su sustentabilidad y acumulación de experiencia (Waissbluth et al., 2014).

Por otra parte, a lo largo de Chile existen muchos subsistemas que se replican en cada región, donde articular procesos de colaboración interinstitucional permitiría probar soluciones innovadores que, posteriormente, podrían ser replicadas y escaladas con alcance nacional. Basta considerar, solo a modo de ejemplo, lo que acontece en los procesos de compras de los municipios de Chile. En cada región hay decenas de municipios, cuyos procesos de abastecimiento son auditados por una Contraloría Regional, y apoyados constantemente por Centros ChileCompra ${ }^{7}$. Si se articulasen procesos de colaboración inter-institucional al interior de cada uno de estos subsistemas, es altamente probable que se genere un sinfín de aprendizajes que podrían servir para el diseño de nuevas políticas de compras del Estado a nivel municipal que permitan mejorar los niveles actuales de probidad, transparencia, eficacia y eficiencia actual.

Lo anterior solo hace referencias de cómo puede aportar la colaboración inter-institucional en el diseño e implementación de nuevas soluciones a problemas públicos. Su alcance no se acota, en ningún caso, solo a esto: recordar que en países nórdicos como Dinamarca, Finlandia, Islandia, Noruega y Suecia, entre un $70 \%$ y $80 \%$ de los organismos públicos que han innovado lo han hecho adoptando y adaptando la experiencia de otras organizaciones, tanto públicas como privadas (Bloch y Bugge, 2013).

\section{Recomendaciones para estudios futu- ros}

Los procesos de innovación colaborativa se centran en construir soluciones integradoras entre actores que enfrentan problemas comunes en diversos sectores y niveles jerárquicos. Si bien la diversidad favorece la divergencia de ideas y exploración de

\footnotetext{
${ }^{7}$ Los Centros ChileCompra ofrecen servicios de capacitación y asesorías tanto a compradores como proveedores del Estado para apoyar su participación en el mercado público.
} 
oportunidades, también puede constituir una barrera. La mera comprensión de qué se entenderá por innovación puede diferir drásticamente entre la alta dirección de un servicio público y su planta administrativa. $\mathrm{O}$ pueden existir diferencias de tipo geográfico, cultural, religioso, étnico, político y económico entre los actores que pueden impedir un intercambio fluido de información. Así, una instancia que dirija diálogos y concrete acuerdos es fundamental. En esta línea, se recomienda trabajar en modelos metodológicos que permitan estructurar procesos de innovación pública colaborativa de manera amplia, considerando aquellos donde se vincule al sector privado, tercer sector, y la ciudadanía.

Por su parte, el presente trabajo no tuvo entre sus objetivos identificar los factores institucionales que puede llevar a una institución pública a innovar. Son múltiples las hipótesis que pueden ser esbozadas al respecto: contexto político que presiona un cambio, ineficiencia en el uso de recursos y cuestionamiento social, incumplimiento de una norma que garantiza un derecho, nuevo entorno tecnológico que habilita la creación de ideas que antes eran no eran factibles, etc. Así, se propone desarrollar estudios de caso donde se pueda determinar, a través de diversos niveles (por ejemplo, individual, organizacional y entorno) qué factores institucionales pueden explicar el éxito o fracaso de procesos de innovación. Asimismo, sería de gran relevancia tener aproximaciones sobre elementos organizacionales que faciliten a adopción de culturas innovadoras, las cuales pueden desencadenar múltiples procesos de innovación.

Sobre la implementación de las innovaciones, se requiere capacidad para gestionar cambios. Ello se traduce en dedicación y efectividad para que equipos de trabajo, provenientes de diversos sectores y niveles, den cumplimiento a compromisos en contextos burocráticos, rígidos y, muchas veces, desintegrados y desinteresados. Asimismo, un factor de alta complejidad es lidiar con los errores y frustraciones que conlleva innovar. Al respecto, se recomienda trabajar en la búsqueda de evidencia científica que permita contrastar los beneficios de dicho proceso versus los costos del mismo.

Un punto adicional, y que no es abordado en el presente trabajo, es estudiar sobre cómo escalar innovaciones. Dada la heterogeneidad que existe en Chile a nivel político, económico, social y cultural, una solución que funciona en contextos acotados no implica que funcione en cualquier otro. De esta manera, al adoptar una innovación que fue desarrollada "fuera de casa" y que se desea escalar, se recomienda tener en cuenta que se requiere de una fase de adaptación mutua: las innovaciones deben adaptarse a las organizaciones en su propio contex- to, así como las instituciones deben adaptarse al nuevo contexto que genera la innovación.

\section{Referencias}

Bason, C. (2010). Leading Public Sector Innovation: Co-Creating for a Better Society. The Policy Press, Bristol, UK.

Behn, R. D. (2008). The adoption of innovation: The challenge of learning to adapt tacit knowledge. En Innovations in Government: Research, Recognition, and Replication, pp. 138-158. Brookings Institution Press, Washington D.C., EE. UU.

Benington, J. y Moore, M. (2011). Public Value in Complex and Changing Times. En Moore, M., editor, Public value: Theory and practice, pp. 130. Palgrave Macmillan, Gran Bretaña.

Bloch, C. y Bugge, M. M. (2013). Public sector innovation-From theory to measurement. Structural Change and Economic Dynamics, 27:133145 .

Brown, T. (2009). Change by Design: How design thinking transforms organizations and inspires innovation. HarperCollins Publishers, New York, USA.

Centro de Sistemas Públicos (2015). Colaboración Pública: Manual Metodológico para la Innovación en el Sector Público.

De Vries, H., Bekkers, V., y Tummers, L. (2015). Innovation in the public sector: A systematic review and future research agenda. Public administration.

Denner, L. y Diaz, T. (2011). Knowledge management in thepublic sector: an online presence as a tool for capture and sharing.

Eggers, W. y Kumar, S. (2009). The Public Innovator's Playbook: Nurturing Bold Ideas in Government. Deloitte Research and the Ash Institute for Democratic Governance and Innovation at Harvard Kennedy School of Government, Winnipeg, MB, Canadá.

European Commission (2013). Powering European Public Sector Innovation: Towards A New Architecture.

Farah, M. y Spink, P. (2008). Subnational Government Innovation in a Comparative Perspective: Brazil. En Borins, S., editor, Innovations in government: Research, recognition, and replication, pp. 71-112. Brookings Institution Press, Washington, DC. 
Gassmann, O. y Schweitzer, F. (2014). Managing the Unmanageable: The Fuzzy Front End of Innovation. En Gassmann, O. y Schweitzer, F., editores, Management of the Fuzzy Front End of Innovation, pp. 3-14. Springer International $\mathrm{Pu}-$ blishing.

Gaubinger, K. y Rabl, M. (2014). Structuring the Front End of Innovation. En Gassmann, O. y Schweitzer, F., editores, Management of the Fuzzy Front End of Innovation, pp. 15-30. Springer International Publishing.

Hartley, J. (2008). Does Innovation Lead to Improvement in Public Services? Lessons from the Beacon Scheme in the United Kingdom. En Borins, S., editor, Innovations in Government: Research, Recognition, and Replication, pp. 159-187. Brookins Institution Press, Washington, DC.

Hartley, J., Sørensen, E., y Torfing, J. (2013). Collaborative Innovation: A Viable Alternative to Market Competition and Organizational Entrepreneurship. Public Administration Review, 73(6):821-830.

Herstatt, C. y Verworn, B. (2001). The "Fuzzy Front End. of Innovation (Working Paper No. 4).

IDEO (2011). Innovation in Government.

Kattel, R., Cepilovs, A., Drechsler, W., Kalvet, T., Lember, V., y Tõnurist, P. (2013). Can we measure public sector innovation? A literature review. LIPSE Working Papers, (no. 2).

Kim, J. y Wilemon, D. (2010). Accelerating the fuzzy front-end of NPD projects: methods and management. International Journal of Engineering Management and Economics, 1(1):80-101.

Koen, P., Ajamian, G., Burkart, R., Clamen, A., Davidson, J., D'Amore, R., Elkins, C., Herald, K., Incorvia, M., Johnson, A., Karol, R., Seibert, R., Slavejkov, A., y Wagner, K. (2001) Providing Clarity and a Common Language to the "Fuzzy Front End". Research-Technology Management, $44(2): 46-55$.
Ministerio de Salud (2011). Encuesta Nacional de Salud ENS Chile 2009-2010.

Mootee, I. (2011). Strategic Innovation and The Fuzzy Front End.

Mulgan, G. (2007). Ready or not? Taking innovation in the public sector seriously. NESTA.

Mulgan, G. y Albury, D. (2003). Innovation in the Public Sector. Technical report, London, UK.

Puttick, R. y Ludlow, J. (2013). Standards of Evidence: An approach that balances the need for evidence with innovation.

Reid, S. y de Brentani, U. (2004). The Fuzzy Front End of New Product Development for Discontinuous Innovations: A Theoretical Model. The Journal of Product Innovation Management, 21(3):170-184.

Seguel, G. (2013). ¿Por qué debemos preocuparnos del pie diabético? Importancia del pie diabético. Revista Médica de Chile, 141(11):1464-1469.

Sørensen, E. y Torfing, J. (2011). Enhancing Collaborative Innovation in the Public Sector. Administration \& Society, 43:842-868.

Verworn, B. (2009). A structural equation model of the impact of the fuzzy front end on the success of new product development. Research Policy, 38(10):1571-1581.

Waissbluth, M. (2008). Sistemas complejos y gestión pública. Documentos de Trabajo, Serie de Gestión, Departamento de Ingeniería Industrial, Universidad de Chile.

Waissbluth, M., Galaz, P., Aguilera, I., Infante, A., Inostroza, J., y Barros, A. (2014). Colaboración Pública: Sistema de gestión del conocimiento para la materialización de innovaciones co-creadas en gobiernos locales. En 5to Congreso de la Sociedad Chilena de Políticas Públicas, Santiago. 